Cahiers d'études italiennes

18 | 2014

NOVECENTO... E DINTORNI

Da Torino a Parigi: Laura Malvano storica e critica d'arte

\title{
Tre diverse concezioni del patrimonio culturale
}

Trois manières de concevoir le patrimoine culturel

Three Different Ways of Conceiving the Cultural Heritage

Valentino Baldacci

(2) OpenEdition

Journals

Edizione digitale

URL: http://journals.openedition.org/cei/1518

DOI: $10.4000 /$ cei. 1518

ISSN: 2260-779X

Editore

UGA Éditions/Université Grenoble Alpes

Edizione cartacea

Data di pubblicazione: 30 mars 2014

Paginazione: $47-59$

ISBN: 978-2-84310-268-4

ISSN: $1770-9571$

Notizia bibliografica digitale

Valentino Baldacci, «Tre diverse concezioni del patrimonio culturale», Cahiers d'études italiennes

[Online], 18 | 2014, online dal 30 septembre 2015, consultato il 26 mars 2021. URL: http:// journals.openedition.org/cei/1518; DOI: https://doi.org/10.4000/cei.1518 


\title{
TRE DIVERSE CONCEZIONI \\ DEL PATRIMONIO CULTURALE
}

\author{
Valentino Baldacci
}

\section{Il primato dell'estetica e la moltiplicazione delle categorie}

Coesistono nel nostro tempo tre diverse concezioni di patrimonio culturale. Si prenda il caso dell'Italia alla quale ci riferiremo nelle linee che seguono soprattutto allorché saranno evocati gli aspetti legislativi e istituzionali della questione. La prima concezione si riferisce alle singole tipologie di beni culturali. Il "Codice dei beni culturali e del paesaggio» ne individua alcune: cose di interesse artistico, storico, archeologico, etnoantropologico, archivistico e bibliografico; ma, come vedremo più avanti, lascia aperta la strada all'inclusione di altre tipologie.

Va notato che questa tassonomia è andata variando nel tempo; anzi, l'analisi di queste variazioni dà preziose indicazioni sul variare della cultura egemone in ciascuna epoca storica. Se prendiamo il più importante documento normativo sulla tutela precedente il Codice attualmente in vigore, cioè le due leggi del 1939, promosse dall'allora Ministro per l'Educazione nazionale Giuseppe Bottai ${ }^{\mathrm{I}}$, già dai titoli si ricava che venivano prese in considerazione soltanto «le cose d'interesse artistico e storico» e «le bellezze naturali».

È vero che, scorrendo l'art. I della L. no I089, si trovavano elencate, oltre a quelle già citate, anche le «cose» che presentavano interesse archeologico o etnografico, precisando ulteriormente che in queste categorie erano comprese anche le «cose» che interessavano la paleontologia, la preistoria e le primitive civiltà; quelle d'interesse numismatico; i manoscritti, gli autografi, i carteggi, i documenti notevoli, gli incunaboli, nonché i libri, le stampe e le incisioni aventi carattere di rarità e di pregio. Infine

I. Legge $\mathrm{n}^{\circ}$ I089 del I giugno I939: Tutela delle cose d'interesse artistico e storico; Legge $\mathrm{n}^{\circ}$ I497 del 9 giugno 1939: Protezione delle bellezze naturali. 
si precisava ulteriormente che vi erano pure comprese le ville, i parchi e i giardini che avessero interesse artistico o storico.

Il progressivo ampliamento delle categorie comprese nella legge di tutela non toglieva che fra le tipologie elencate sussistesse un rapporto gerarchico d'importanza, sottolineato appunto dal titolo stesso della legge: le «cose» tutelate in quanto patrimonio culturale lo erano soltanto in quanto avevano un interesse artistico o storico: l'elencazione delle altre tipologie serviva soltanto a chiarire le intenzioni del legislatore. Così i beni archeologici erano tali solo se rientravano in queste due categorie primarie, e a maggior ragione quelli etnografici; le ulteriori precisazioni rispetto alla paleontologia e alla preistoria appaiono pleonastiche; a maggior ragione gli archivi e le biblioteche erano prese in considerazione sempre e soltanto in rapporto al loro valore di raccolte di documenti storici. Surrettiziamente, quasi per inciso, venivano inseriti quelli che oggi chiamiamo beni architettonici, però solo sotto la specie di ville, parchi e giardini, sempre che, beninteso, rispettassero i due canoni fondamentali.

Se poi si prende in considerazione l'altra legge promossa da Bottai in quel 1939 («Protezione delle bellezze naturali»), anche in questo caso il titolo è estremamente chiarificatore: non il paesaggio, come in seguito è stato definito, ma solo le bellezze naturali sono tutelate. È cioè il valore estetico, e quindi l'eccezionalità, la discriminante fondamentale. Questa discriminante era fortemente presente anche nella legge $n^{\circ}$ I089 perché, se è vero che varie categorie di beni erano tutelati in virtù del loro valore storico, era prevalente quello estetico, che non solo restava l'unico valido per i beni artistici, così esplicitamente richiamati, ma proiettava la sua influenza anche su altre categorie, e in particolare sulle architetture.

Si veniva così a creare una sorta di gerarchia fra i beni culturali, che vedeva al primo posto quelli artistici, portatori dei fondamentali valori estetici; seguivano quelli sui quali in qualche modo viene proiettata, sia pure in modo indiretto, la luce proveniente dai primi, quindi le architetture e i reperti archeologici. Il resto, cioè documenti d'archivio (purché «notevoli») e i libri aventi carattere di rarità e di pregio, era tutelato solo in quanto si riferiva a una documentazione storica di particolare importanza; anzi, in quella espressione "di pregio» riappariva, anche qui, l'importanza primaria del valore estetico. L'inserimento fra i beni da tutelare di quelli di interesse etnografico, singolarmente connessi a quelli archeologici, appariva più un relitto ottocentesco che un'apertura a una nuova sensibilità.

Tutto ciò è ben noto e più volte sottolineato dalla letteratura sull'argomento. Se ha un senso tornarci sopra e sottolinearlo è perché, nonostante il tempo trascorso dal 1939 e i grandi mutamenti politici, istituzionali e 
culturali intervenuti, questo impianto culturale, e in particolare l'idea di una gerarchia fra i beni culturali imperniata sul primato dei valori estetici, è ancora largamente presente e anzi, si potrebbe dire, ancora dominante, avendo ricevuto un insospettato aiuto dalla diffusione della cultura di massa e dal turismo culturale, cioè proprio da quei fenomeni che la cultura idealistica, che sta alla base delle leggi del 1939, non avrebbe certo visto di buon occhio. Il fatto è che l'industria culturale, comprendendo in essa non soltanto l'editoria ma soprattutto la televisione e la fabbrica di mostre, eventi culturali e simili, ha contribuito in maniera decisiva a trasformare le opere d'arte e i relativi autori in feticci, in oggetti e figure mitiche, da adorare in forme cultuali indipendentemente dalla reale comprensione delle opere e degli autori stessi. Questo fenomeno ha trasferito a livello di massa l'idea della gerarchia dei beni culturali e quella del primato dell'opera d'arte, divenuta oggetto di culto privo di ogni riferimento critico e contestualizzante e ricondotto soltanto a momenti e situazioni puramente emozionali (da qui, per inciso, l'enorme successo, che non accenna a calare, della pittura impressionistica).

C'è un altro aspetto che merita di essere sottolineato. Ricondurre la definizione di patrimonio culturale alla particolare tipologia di appartenenza della cosa tutelata comporta la perdita di una definizione complessiva di patrimonio culturale e l'introduzione, non casuale, dell'espressione plurale «beni culturali». Quella che viene così messa in evidenza non è una definizione unitaria di patrimonio culturale ma quella particolare relativa a ciascuna tipologia: si avrà così una definizione di bene artistico, una di bene archeologico, ecc. Anche quando il ricordato primato della dimensione estetica comincerà, con il mutamento dell'egemonia culturale, a declinare, resterà in piedi, e in larga misura è ancora fortemente vigente, l'idea di una frammentazione del patrimonio culturale nelle singole tipologie nelle quali lo si vuol classificare. Si afferma così una impostazione tassonomica che vede, accanto al prevalere della cultura idealistica espressa nell'idea del primato dell'estetica, la coesistenza di vecchie categorie risalenti alla cultura positivistica.

\section{Una concezione identitaria di patrimonio culturale}

La seconda concezione si esprime nel recupero di una concezione unitaria del patrimonio culturale (si usa appunto l'espressione "patrimonio culturale» e non quella di «beni culturali») e soprattutto in una diversa concezione che mette al centro il suo significato identitario. Il patrimonio 
culturale è così visto, indipendentemente dalla sua articolazione in varie tipologie e dal suo valore estetico, come espressione (l'espressione più alta, se vogliamo) dell'identità di una comunità: una comunità che può avere una dimensione più o meno ampia — dalla comunità locale a quella nazionale fino a quella comunità che è costituita dall'intera umanità.

Almeno in Italia questa concezione si è manifestata abbastanza precocemente, prima ancora della creazione del Ministero dei Beni Culturali e Ambientali. Il riferimento d'obbligo è al documento prodotto al termine dei suoi lavori, nel 1966, dalla Commissione parlamentare (istituita con Legge 26 aprile 1964, $\mathrm{n}^{\circ}$ 310) presieduta dall'on. Francesco Franceschini, che definì bene culturale «tutto ciò che costituisce testimonianza materiale avente valore di civiltà». Come si vede, venivano del tutto abbandonate la definizione pluralistica sulla base delle tipologie e il principio del primato del valore estetico e si metteva al centro della nuova definizione unitaria quello della testimonianza di civiltà. Questa definizione non solo affermava un altro primato, quello del documento storico, ma apriva la strada all'affermazione del valore del patrimonio culturale come aspetto essenziale dell'identità di una comunità. Il concetto di identità resta a tutt'oggi assai discusso, perché da parte di alcuni esso viene rifiutato sia in base a considerazioni di carattere politico, che fanno riferimento alle degenerazioni di carattere nazionalistico che, in passato e nel presente, esso avrebbe prodotto; sia anche in base a considerazioni più generali, che hanno alla base la tesi della necessaria «contaminazione» (spesso si adopera addirittura il termine di «meticciato», con significato positivo rispetto a quello, più negativo, tradizionale) fra culture e popoli, e quindi comunità, diverse. Considerazioni, come si vede, più generali, ma altrettanto "politiche», o addirittura ideologiche, rispetto alle precedenti.

A parte la riflessione sui possibili esiti degenerativi di questo principio, che affronteremo a parte, resta il fatto che l'idea del patrimonio culturale come testimonianza di civiltà e quindi, conseguentemente, come fattore di identità di una comunità, ha fatto molta strada. È stata accolta, a livello nazionale, nel Codice dei beni culturali e del paesaggio, dove all'art. 2 comma 2, in maniera piuttosto confusa, in un primo tempo si conserva l'espressione plurale «beni culturali», elencandone le tipologie in base all'interesse artistico, storico, archeologico, etno-antropologico, archivistico e bibliografico; ma poi si introduce una nozione residuale, che in realtà finisce per essere universalmente definitoria, parlando di «testimonianze aventi valore di civiltà». Ma più significativo e chiaro è l'art. I comma 2 dove la funzione del patrimonio culturale è individuata nella preservazione della «memoria della comunità nazionale e del suo territorio». Va 
ricordato che, nei lavori preparatori del Codice, la Commissione cultura della Camera dei Deputati aveva definito il patrimonio culturale come "elemento costitutivo e rappresentativo dell'identità nazionale», definizione poi superata nel testo definitivo.

Anche a livello internazionale ci si è mossi nella stessa direzione. La stessa nozione di «Patrimonio universale dell'umanità», elaborata dall'Unesco e che sta conoscendo una grande fortuna, va nello stesso senso, assumendo l'intera umanità come comunità universale, nel senso già ricordato sopra, e che ovviamente non nega l'esistenza di altre comunità particolari. Anche l'ICOM (International Council of Museums) accoglie nel suo statuto la definizione di «testimonianze materiali e immateriali dell'umanità».

Proprio l'emergere di istanze universalistiche e lo stesso crescente sviluppo della globalizzazione in ogni campo, da quello economico a quello della comunicazione, ha fatto crescere l'esigenza di una sottolineatura degli aspetti identitari che caratterizzano una comunità, anche, e forse soprattutto, a livello locale. È degno di nota il fatto che questa esigenza, presente in ogni luogo e in ogni tempo, abbia assunto in questa fase storica la forma della valorizzazione del patrimonio culturale. In altri tempi questa esigenza si esprimeva in modi e con simboli assai diversi: la bandiera e l'inno nazionale; il culto del sangue dei martiri, religiosi o politici poco importa; l'idea della superiorità razziale e antropologica; quella della «necessità storica» della vittoria di una classe sociale con l'eliminazione di tutte le altre, e via elencando.

A proposito dei possibili aspetti degenerativi del principio del patrimonio culturale come rappresentativo dell'identità nazionale, c'è da rilevare come sia venuto modificandosi l'atteggiamento dei belligeranti nei casi di conflitto. In passato, fino alla seconda guerra mondiale, prevaleva un atteggiamento che tendeva a trascurare il significato simbolico del patrimonio culturale a favore di considerazioni puramente militari, appena temperate, in alcuni casi, da valutazioni e da riflessioni di carattere culturale e talvolta politico. In altre parole, i bombardamenti e le distruzioni massicce operate durante la seconda guerra mondiale dai tedeschi sulle città inglesi e dagli anglo-americani sulle città tedesche muovevano da intenti puramente militari, fra i quali, oltre alla distruzione della capacità economica del nemico, anche quella di fiaccare la volontà di combattere e di resistere della popolazione civile. Non sembra invece che si mirasse al patrimonio culturale con particolare intenzione, né di distruggerlo né di preservarlo, a meno che, come si è detto, non intervenissero particolari considerazioni. Il Duomo di Colonia o il centro storico di Dresda non furono distrutti perché questi monumenti assumevano un particolare 
valore simbolico; semplicemente rientravano nella tattica di distruzione integrale delle città tedesche che gli anglo-americani perseguivano così come qualche anno prima avevano fatto i tedeschi con le città inglesi. In certi casi, come per le città d'arte italiane, Roma in primo luogo, intervenivano, a risparmiarle, anche se parzialmente, considerazioni di carattere politico: le ripercussioni sull'opinione pubblica dei propri paesi, o su quella cattolica in particolare, ecc.

In tempi più recenti invece, e con particolare riferimento alle guerre balcaniche, il patrimonio culturale è stato letto e trattato proprio in funzione del suo significato simbolico: il ponte di Mostar fu distrutto dai croati non per esigenze militari ma perché rappresentava un simbolo delle tradizioni locali, di derivazione turco-islamica, ed era al tempo stesso un simbolo di pace e di incontro fra diverse civiltà, che in quel momento veniva rifiutato in nome della lotta all'ultimo sangue fra etnie nemiche. Ancora più di recente, per la Serbia è «impossibile» riconoscere l'indipendenza del Kossovo, abitato per il 90\% da una popolazione albanese, perché in questa regione si trovano i monumenti, in particolare monasteri, che stanno alla base della storia e dell'identità serba.

Tuttavia sarebbe un errore ricavare da questi casi il rifiuto del patrimonio culturale come segno di identità. Anzi, il fatto di assumere il patrimonio culturale come simbolo dell'identità locale o nazionale, invece di altri simboli necessariamente antagonistici come quelli sopra citati, apre la strada al riconoscimento reciproco del valore del patrimonio culturale altrui e quindi delle diverse identità. Nessun simbolo come il patrimonio culturale ha una così forte valenza universale.

\section{La valenza mercantile del patrimonio culturale}

La terza concezione si riferisce al valore economico del bene culturale, insistendo proprio su quell'aspetto sottinteso nel sostantivo «bene» o "patrimonio», indipendentemente dall'aggettivo.

Questa dimensione economica può essere presa in considerazione secondo varie modalità. La prima, e la più ovvia, si riferisce proprio al valore monetario, di mercato, del bene preso in considerazione. Le cronache sono piene di casi di opere d'arte (ma talvolta anche di strumenti musicali rari, oppure di libri altrettanto rari) venduti all'asta per cifre altissime. Questi episodi inducono nell'opinione pubblica l'idea che ci sia un legame tra il valore monetario e quello artistico dell'opera, laddove è evidente che il primo è soggetto alle norme che regolano lo scambio delle merci sul mer- 
cato (rarità, utilità, ma anche gusto, moda ecc.), mentre il secondo segue regole sue proprie, anch'esse soggette a variazioni ma secondo parametri propri.

C'è da rilevare che grandi variazioni sono intervenute nel mercato dei beni culturali a partire dagli inizi del XX secolo. In precedenza, in un'epoca dominata dall'ideologia liberista, le opere d'arte, i reperti archeologici, ecc., venivano comprati e venduti in quasi totale libertà: erano considerati beni più o meno come gli altri, e solo in determinati e rari casi (monumenti nazionali) la loro vendita era soggetta a restrizioni. A partire dall'inizio del XX secolo si diffuse l'idea che il patrimonio culturale era fortemente connesso all'idea di identità nazionale, e che quindi occorreva regolarne lo scambio economico, in particolare era necessario restringerne l'esportazione all'estero. Nacquero così in Italia, ma anche negli altri paesi, normative vincolistiche: la prima per il nostro paese, è la Legge $n^{\circ}{ }^{2} 85$ del I2 giugno 1902 (Legge Nasi) che pose un freno, anche se non assoluto, all'esportazione delle opere d'arte.

La legislazione vincolistica si è andata progressivamente inasprendo, anche se non è mai stato posto un divieto assoluto alla commerciabilità dei vari tipi di beni, specialmente verso l'estero. L'istituto della notifica, introdotto nel 1909, ha comunque posto sotto controllo il commercio delle opere considerate di interesse artistico e storico. Questi vincoli hanno fatto sì che per determinate tipologie di patrimonio culturale (in particolare per le architetture) la dichiarazione di interesse culturale finisse per abbassare notevolmente il suo valore di mercato.

Ma quello del valore del singolo bene è solo un aspetto della dimensione economica del patrimonio culturale, e nemmeno il più importante. Maggiore rilevanza hanno tutte quelle attività che sono in qualche modo connesse al patrimonio culturale e che si è soliti indicare con il termine di indotto. La più visibile di queste attività è certamente il turismo culturale, che è venuto assumendo negli ultimi decenni proporzioni gigantesche. All'antica pratica del grand tour, riservata ai giovani delle famiglie aristocratiche e improntata a motivi di studio e di educazione, si è sostituito un gigantesco intreccio di spostamenti e di viaggi che coprono tutto il pianeta. Le tipologie del turismo culturale sono numerose e in continua evoluzione. La più tradizionale è quella riferita alle città d'arte, i centri che in Europa, e in particolare in Italia, raccolgono i monumenti più noti e i musei più famosi. Ma negli ultimi anni si è sviluppato un turismo culturale che si indirizza in due direzioni opposte: da un lato c'è stata la progressiva scoperta del patrimonio culturale «minore», rappresentato da borghi, cittadine, castelli, conventi e monasteri isolati, in precedenza 
appena sfiorati dal turismo culturale. In questa tipologia di turismo un ruolo fondamentale è svolto dal paesaggio, che sempre più è oggetto di particolare attenzione da parte di turisti il cui obiettivo è la ricerca di un ambiente diverso da quello standardizzato delle città di residenza. Si tratta di un turismo che si dirige prevalentemente, anche se non unicamente, verso aree e luoghi non lontani dalla località di residenza, che possono essere raggiunti nel corso di una sola giornata o al massimo di un week-end. $\mathrm{Al}$ polo opposto si colloca la crescente scoperta di aree e località esotiche, poste in paesi anche lontanissimi da quello di residenza. Questo tipo di turismo mette in moto, naturalmente, spostamenti di notevoli dimensioni e richiede un impiego di tempo (e di denaro) piuttosto elevato. Alla base della crescita progressiva di questo tipo di turismo c'è da un lato, da parte di un certo tipo di pubblico, l'esaurimento delle mète più tradizionali; dall'altro la scoperta che il patrimonio culturale non è presente soltanto nelle zone di più antica e tradizionale civilizzazione, in particolare in Europa, ma può essere scoperto in aree che fino a non molto tempo fa erano mète soltanto di tipologie di turismo molto particolari, ristrette a gruppi di persone limitati, come i cultori dei safari, ecc. Naturalmente ha concorso a questa diffusione di un turismo culturale verso mète lontane il progressivo abbassamento dei prezzi, soprattutto quelli dei viaggi aerei, connesso prima alla pratica dei voli charter e poi di quelli low cost. $\mathrm{E}$ appena il caso di sottolineare quali e quanti settori economici sono messi in movimento dalle tipologie di turismo culturale sopra indicate. Si va dai trasporti (aerei, ferroviari, marittimi, automobilistici) a tutta la rete dell'accoglienza e dell'ospitalità, dagli alberghi ai ristoranti, dai bar agli agriturismo. Oltre alle attività specializzate, come quelle delle agenzie di viaggio e a quelle delle guide, è poi tutta la rete commerciale delle città e delle zone interessate che viene coinvolta, in quanto il turismo esprime una domanda aggiuntiva rispetto a quella abituale dei residenti; anzi è ben noto che esiste una maggiore propensione al consumo quando si è fuori della propria area abituale di residenza.

A parte va considerata l'attività di quelle strutture che, per loro natura, sono direttamente connesse al patrimonio culturale: i musei innanzi tutto, e poi l'organizzazione delle mostre. Musei e mostre vanno considerate da un lato come occasioni aggiuntive di richiamo rispetto a quello offerte dai monumenti e dal paesaggio, dall'altro come strutture autonome che sviluppano una loro attività che ha una notevole rilevanza economica.

Un museo va considerato, oltre che come luogo di conservazione e di esposizione di collezioni e centro di attività culturale, come soggetto di una molteplicità di attività economiche che lo rendono per certi aspetti 
simile a un'azienda commerciale, con i suoi costi e ricavi, le sue problematiche manageriali, ecc. Se i costi di un museo sono identificabili in quelli che normalmente figurano in un'attività commerciale (personale, energia, ecc.), a questi vanno aggiunti quelli che mirano alla conservazione e al restauro delle opere. I ricavi sono di molteplice natura: prima di tutto la bigliettazione, che può assumere le forme più raffinate e complesse; poi l'associazione al museo (membership) che tende alla fidelizzazione del visitatore; inoltre l'affitto degli strumenti di informazione, laddove non siano gratuiti (audioguide, ecc.). Una voce rilevante è costituita dallo store (o bookshop, come viene impropriamente chiamato in Italia), che nei grandi musei raggiunge non solo notevole dimensioni ma anche livelli di qualità assai elevati. Ci sono poi ricavi derivanti dalle vere e proprie attività culturali: visite guidate, conferenze, lezioni, concerti, cicli di film, attività teatrali. In alcuni casi i musei si fanno promotori e organizzatori di viaggi all'estero verso località considerate di particolare attrattività culturale. Un'altra fonte di introito è costituita dai ristoranti e caffetterie interni al museo, sia in forma di gestione diretta che di diritti di concessione. Sempre più spesso gli spazi dei musei vengono offerti a pagamento per occasioni di vario genere, dalle cene aziendali alle sfilate di moda. Un'altra voce significativa delle entrate è costituita dalle donazioni e dalle offerte volontarie, che spesso sono organizzate e sollecitate in forma assai elaborata. Infine, soprattutto nei musei europei, è fondamentale l'apporto costituito dai contributi provenienti dagli enti pubblici, siano essi lo Stato, le Regioni o gli enti locali. Al di là del dibattito sulla possibilità che un museo possa essere gestito in pareggio per mezzo delle sole risorse proprie, resta il fatto che la gestione di un museo, oltre a una dimensione culturale, ne presenta una strettamente manageriale.

Per le mostre, valgono in larga misura riflessioni simili, con alcuni aspetti, relativi ai costi e ai ricavi, analoghi ed altri specifici. Per i costi, ovviamente una voce di particolare rilievo è costituita dai trasporti, dalle assicurazioni e dall'allestimento temporaneo, nonché dalle spese per promozione e pubblicità. Per i ricavi sono meno rilevanti quelli derivanti da un'attività permanente e continuativa come quella di un museo, ma a compensare questi minori introiti stanno quelli derivanti dall'aspetto di eccezionalità che ogni mostra, quale più quale meno, assume; e quindi maggiore affluenza di visitatori, maggiore numero di biglietti venduti, maggiore vendita di tutta la produzione accessoria, come i cataloghi, i poster, l'oggettistica e in generale il merchandising, maggiore occasione di entrate derivanti da sponsorizzazioni. 
Se il turismo culturale e la gestione dei musei e delle mostre sono gli aspetti più visibili della dimensione economica del patrimonio culturale, non si devono dimenticare altre attività che, pur meno evidenti, tuttavia hanno anch'esse ricadute economiche non trascurabili. Una dimensione economica l'ha la stessa attività di catalogazione del patrimonio culturale, svolta in Italia dalle Soprintendenze ma che coinvolge anche soggetti esterni ad esse, come, ad esempio, gli esperti in elaborazione di programmi informatici e anche gli stessi compilatori di schede. Un'attività che in alcune realtà ha assunto un rilevante significato economico è quella del restauro. Il restauro, sia pittorico e plastico che architettonico, ha da tempo superato lo stadio artigianale nel quale era prevalente l'abilità manuale del restauratore nonché ovviamente la sua preparazione storico-artistica, per utilizzare tecniche informatiche di elevato contenuto tecnologico. Ci riferiamo non solo all'esecuzione del restauro ma anche e soprattutto alle analisi diagnostiche che precedono il restauro stesso e che ormai coinvolgono competenze di altissimo livello.

\section{Conclusione}

Ognuna di queste tre dimensioni del patrimonio culturale ha una sua validità e una sua legittimità. Si pone tuttavia il problema del loro rapporto reciproco e soprattutto quello di possibili collisioni e contraddizioni, a seconda che si privilegi l'una o l'altra.

Viene voglia di scomodare Platone, che distingueva le tre forme legittime di governo (monarchia, aristocrazia, democrazia) dalle loro degenerazioni (tirannide, oligarchia, demagogia). Anche le tre dimensioni del patrimonio culturale, ognuna delle quali ha in sé la sua legittimità, possono degenerare, soprattutto se intese unilateralmente, senza tener conto delle altre due.

Così è evidente che assumere la dimensione economica come la sola, o comunque la prevalente, nel guidare le scelte della politica dei beni culturali e la gestione delle strutture ad essi inerenti porta a gravi degenerazioni, che hanno come conseguenza il rischio del depauperamento dello stesso patrimonio. Ciò è particolarmente evidente nel caso del paesaggio, dove uno sfruttamento senza regole del medesimo porta alla conseguenza, purtroppo ben visibile in un notevole numero di casi, della perdita o comunque del degrado proprio di quei valori paesaggistici che si volevano sfruttare. 
Anche la definizione identitaria del patrimonio culturale può portare a degenerazioni. Ne abbiamo già visto alcuni esempi, ma più in generale è la dimensione stessa dell'identità che può condurre a gravi degenerazioni. La storia degli ultimi due secoli è purtroppo ricca di insegnamenti in questo senso, partendo dalle spoliazioni compiute dai francesi in età napoleonica a danno dei paesi occupati agli esempi sopra citati, ai quali altri si potrebbero aggiungere. Il sentimento dell'identità, irrinunciabile in ogni persona, può condurre all'egoismo e all'insensibilità verso il prossimo. Così per i popoli o le comunità: il diritto a coltivare e a difendere la propria identità può trasformarsi in chiusura o addirittura in ostilità verso le identità altrui. D'altra parte abbiamo già detto che le identità fondate sul patrimonio culturale sono le più aperte, le più disponibili ad accogliere i valori identitari di altre comunità, perché il patrimonio culturale è meno disponibile di altre forme simboliche ad essere letto attraverso i moduli dell'intolleranza e della chiusura, e rinvia invece a forme universali, sia pure comprese nei loro contesti storici.

I rischi inerenti alle dimensioni economica e identitaria del patrimonio culturale sono abbastanza evidenti e quindi è più agevole prendere le necessarie contromisure. Meno evidente e più sottile è il rischio legato a un'altra unilateralità, quella di assumere come esclusiva la dimensione inerente a ciascuna tipologia di patrimonio culturale. È più sottile perché è evidente la legittimità di assumere come criterio di valutazione di ciascuna tipologia criteri elaborati all'interno della tipologia stessa. Ogni ramo del sapere e della conoscenza ha un suo proprio statuto ed è questo statuto che consente il dialogo fra competenze e fra saperi.

Il problema nasce quando si pretende di imporre questo statuto anche a dimensioni del patrimonio culturale che sono esterni alla logica propria di ciascuna tipologia. Che gli storici dell'arte elaborino una loro propria teoria relativa non solo agli aspetti estetici ma anche alle tecniche della conservazione e del restauro non solo è legittimo ma è del tutto necessario. Ma non è più legittimo pretendere che questa dimensione interna alla cultura storico-artistica sia l'unica possibile e, in nome di questo esclusivismo, rifiutare di prendere in considerazione la dimensione identitaria del patrimonio culturale o addirittura rifiutare in partenza ogni considerazione della dimensione economica dello stesso.

Il rifiuto della dimensione identitaria porta alla conseguenza di considerare il patrimonio culturale come un affare esclusivo degli addetti ai lavori, intendendo soltanto gli specialisti di una determinata disciplina, e a trascurare quindi la dimensione del pubblico, cioè dei cittadini, dell'intera comunità, che, anche se privi di conoscenze specialistiche, sono fortemente 
interessati al patrimonio culturale e alla sua conoscenza. Di fatto questa cultura porta a un atteggiamento di tipo elitario, che nega di fatto ogni validità alla dimensione educativa, alla diffusione fra i cittadini della conoscenza del patrimonio culturale, intesa appunto come patrimonio comune di tutta la comunità. Porta anche, come ulteriore conseguenza, a considerare validi sul piano scientifico soltanto quei rami del sapere interni alla tipologia considerata e trascurare altri saperi (per esempio, la sociologia, la psicologia, la scienza della comunicazione) che possono dare un valido aiuto alla diffusione della conoscenza del patrimonio culturale.

Più drastico ancora, e più carico di significati ideologici, è il rifiuto di accettare la dimensione economica del patrimonio culturale, perché si accompagna a una serie di tesi legate al rifiuto della mercificazione del sapere, del condizionamento capitalistico non solo della produzione economica ma anche dell'espressione culturale e altri simili rifiuti, che si basano su una concezione antagonistica della società contemporanea che viene rifiutata in toto in quanto condizionata e succube della forma di produzione capitalistica. Colpisce il fatto che una simile cultura, ereditata dal periodo dell'egemonia culturale del marxismo, sia ridotta a dimensioni minoritarie in qualunque altro campo, salvo, forse, in quello del patrimonio culturale, nel quale la difesa contro ogni forma di inquinamento capitalistico si sposa strettamente con la difesa della sua purezza, eredità di un'epoca precapitalistica.

Aspetti positivi e aspetti negativi di questi rifiuti si intrecciano in maniera che appare indissolubile. Per orientarsi in questo che appare un labirinto inestricabile occorre assumere un atteggiamento fortemente pragmatico, che si appoggi da un lato ad alcuni principi ma che rifiuti anche ogni pregiudiziale di carattere ideologico. I principi che appaiono irrinunciabili sono abbastanza evidenti e alla fine si riducono a uno: che ogni uso del patrimonio culturale non comporti il deterioramento e il degrado del medesimo. Fatto salvo questo principio, ogni altro tipo di azione dovrà essere esaminata e valutata sulla base di orientamenti pragmatici, che tengano conto di tutte e tre le dimensioni sopra enunciate. Occorre che fra studiosi e operatori che agiscono nelle tre dimensioni messe in evidenza si crei un clima basato sullo sforzo di comprendere le ragioni degli altri, anche se sono lontane dall'impostazione culturale nella quale si sono formati. È difficile per lo storico dell'arte e per il funzionario di Soprintendenza accettare che esiste anche una dimensione economica del patrimonio culturale che va tenuta presente e non demonizzata a priori, anche se le ragioni della tutela devono sempre essere considerate prioritarie. È difficile per un operatore economico che agisce nell'ambito dei beni culturali compren- 
dere talvolta le ragioni degli addetti alla tutela, che sembrano porre inutili pastoie all'esercizio di un'attività da essi considerata legittima. È difficile per entrambe queste categorie, addetti alla tutela e operatori economici, comprendere che esiste un interesse del pubblico, dei cittadini, alla conoscenza del patrimonio culturale come espressione fondamentale dell'identità di una comunità che tende a prescindere sia dalle ragioni tecniche della tutela che da quelle dell'interesse economico.

Solo uscendo da una logica basata sull'esclusivismo delle proprie ragioni, dovuta da un lato all'eredità dell'antica impostazione idealistica, fondata sul principio della gerarchia dei valori e sul primato del valore estetico, dall'altro a una cultura dell'iniziativa economica che tiene scarso conto della specificità del campo del patrimonio culturale, si può andare oltre a una logica che appare ancora fortemente condizionata da pregiudizi ideologici o all'opposto da mancanza di principi per approdare a una cultura pragmatica, capace di promuovere una collaborazione fra tutti coloro che, a vario titolo, operano nel campo della tutela e della valorizzazione del patrimonio culturale. 